\title{
A NOTE ON AN INTERIOR SOLUTION FOR A FLUID SPHERE OF CONSTANT GRAVITATIONAL MASS DENSITY IN GENERAL RELATIVITY
}

\author{
J. P. SHARMA
}

In this paper it has been obtained the solutions of the field equations for a fluid sphere of constant gravitational mass density.

\section{Introduction}

Schwarzschild interior solution of the field equations for a fluid sphere of constant density $\rho$ is quite well known and is of great importance in general relativity. Recently, in three subsequent papers $(1972,1973,1976)$, the author has studied planetary structures in general relativity, in general, and the internal structure of Mars in particular. So far as known to the author, a solution of the field equations for a fluid sphere (which is in hydrostatic equilibrium) of constant gravitational mass density $\rho c^{2}+3 P=H$. (1935) has not been attempted. Gravitational attraction of matter is governed by this expression (rather than p). The physical significance of the parameter $H K / 2 \delta^{2}$ has been pointed out.

\section{The Equations of Equilibrium}

In the case of spherical symmetry, the time-independent metric in $(r, \theta, \phi)$ coordinate system is given by (Moller, 1952)

$$
a(r) d r^{2}+r^{2} d \Omega^{2}-b(r) c^{2} d t^{2},
$$

where $d \Omega^{2}=\left(d \theta^{2}+\sin ^{2} \theta d \phi^{2}\right)$. The gravitational equations, needed for our purpose, reduce to

$$
\frac{1}{a r}\left(\frac{1}{b} \frac{d b}{d r}+\frac{1}{r}\right)-\frac{1}{r^{2}}+\lambda=K P
$$

and

$$
\frac{1}{a r}\left(\frac{1}{a} \frac{d a}{d r}-\frac{1}{r}\right)+\frac{1}{r^{2}}-\lambda=K \rho c^{2},
$$

where symbols have their usual significance. In the general theory, the hydrostatic equilibrium equation becomes 


$$
\frac{d P}{d r}+(P+\varepsilon) \frac{1}{2 b} \frac{d b}{d r}=0
$$

where $P$ is the pressure and $\varepsilon=\mathrm{p} c^{2}$ is the energy density written as an equivalent to mass density $\mathrm{p}$ times the square of the speed of light $c^{2}$.

\section{The Desired Solutions}

For a fluid sphere of constant gravitational mass density, our assumed relation, connecting pressure with the energy density, is

$$
\rho c^{2}+3 P=H=\text { constant. }
$$

Substituting (5) into (4), we obtain a solution

$$
H-2 P=\alpha, b,
$$

where $\alpha$ is the constant of integration. Adding (2) and (3), we have

$$
\frac{1}{b} \frac{d b}{d r}+\frac{1}{a} \frac{d b}{d r}=\operatorname{Kar}\left(P+\rho c^{2}\right)
$$

or, in view of equations (5) and (6), this becomes

$$
\frac{d}{d r}(a b)=\operatorname{Kor}(a b)^{2},
$$

the integration of which yields

$$
a b=\frac{2 \beta}{2-K \alpha \beta r^{2}}=\frac{\beta}{1-\delta^{2} r^{2}},
$$

where $\beta$ is another constant of integration and $\delta=(K \alpha \beta / 2)^{\frac{1}{2}}$. Making use of equations (6) and (8) in (2), we obtain

$$
\left(1-\delta^{2} r^{2}\right) r \frac{d b}{d r}+b=\beta+\frac{\beta}{2}(K H-2 \lambda) r^{2} .
$$

Solving this linear differential equation, we obtain

$$
b(r)=\beta\left\{1+L-\frac{L}{\delta r}\left(1-\delta^{2} r^{2}\right)^{\frac{1}{2}} \sin ^{-1} \delta r\right\}+\frac{M}{r}\left(1-\delta^{2} r^{2}\right)^{\frac{1}{2}},
$$

where

$$
L=\frac{1}{2 \delta^{2}}(K H-2 \lambda) ; M=\text { constant }
$$

Since $b(r)$ remains finite at the center $r=0$ of the fluid sphere, $M$ must vanish. Thus equation (10), simply, reduces to 


$$
b(r)=\beta\left\{1+L-\frac{L}{\delta r}\left(1-\delta^{2} r^{2}\right)^{\frac{1}{2}} \sin ^{-1} \delta r\right\} .
$$

Substituting (12) into (8), we get

$$
a(r)=\left\{(1+L)\left(1-\delta^{2} r^{2}\right)-L(\delta r)^{-1}\left(1-\delta^{2} r^{2}\right)^{\frac{3}{2}} \sin ^{-1} \delta\right\}^{-1} .
$$

In view of the above, the product of $K$ and the pressure can be expressed as

$$
K P=\lambda-\delta^{2}+L \delta \frac{\left(1-\delta^{2} r^{2}\right)^{\frac{3}{2}}}{r} \sin ^{-1} \delta r .
$$

The centrál values of $K P$ and $(K \mathrm{p}) c^{2}$ are given by

$$
(K P)_{r=0}=\lambda+(L-1) \delta^{2}=0.5 K H-\delta^{2},
$$

and

$$
(K \rho)_{r=0} c^{2}=3 \delta^{2}-0.5 K H
$$

respectively. These equations make it clear that $K H$ must satisfy the inequality

$$
\delta^{2} \leqslant \frac{K H}{2} \leqslant 3 \delta^{2}
$$

so that $P_{0}$ and $\mathrm{p}_{0}$ remain positive. One may verify that $d P / d r$ is negative, the physical meaning of which is that as the radius increases, the pressure decreases and attains a zero value at the surface boundary $r=r_{1}$ of the sphere, where $x_{1}=\alpha r_{1}$ satisfies

$$
\left(\delta^{2}-\lambda\right) x_{1}=\frac{1}{2}(K H-2 \lambda)\left(1-x_{1}^{2}\right)^{\frac{1}{2}} \sin ^{-1} x_{1},
$$

and we find that it lies between 0 and 1 . At this point we may replace the interior solution by the Schwarzschild exterior solution for which

$$
b(r)=\frac{1}{a(r)}=1-\frac{2 m}{r}-\frac{1}{3} \lambda r^{2} .
$$

For the exterior solution $a(r)$ and $b(r), P$ and $\mathrm{p} c^{2}$ should be continuous everywhere and $a b=1$, therefore, from (8), we find that

$$
\beta=1-\delta^{2} r_{1}^{2} \text {. }
$$

Also, from equations (6) and (19), we find

$$
\frac{H}{\alpha}=\frac{H K \beta}{2 \delta^{2}}=1-\frac{2 m}{r_{1}{ }^{2}}-\frac{1}{3} \lambda{r_{1}}^{2}=\frac{H K}{2 \delta^{2}}\left(\mathrm{i}-\delta^{2}{r_{1}}^{2}\right) .
$$


We may note that $\lambda$ (cosmical constant) has been taken into consideration for the sake of completeness, but in actual situation where the formulae are to be applied we may ignore the same (if not actually zero).

We define the value of the parameter $H K / 2 \delta^{2}$, in terms of the central pressure and central density, as follows :

$$
\frac{H K}{2 \delta^{2}}=\frac{\rho_{0} c^{2}+3 P_{0}}{\rho_{0} c^{2}+P_{0}} .
$$

If we put $\lambda=0$ in $(21)$, then

$$
\frac{2 m}{r_{1}}=1-\left\{\frac{H K}{2 \delta^{2}}\left(1-\delta^{2} r_{1}^{2}\right)\right\} .
$$

Hence, in order that $m$ remains positive,

$$
\frac{H K}{2 \delta^{2}}\left(1-\delta^{2} r_{1}^{2}\right)<1,
$$

which is a strict inequality so as to satisfy the Schwarzschild exterior solution.

\section{R E F E R E N C E S}

['] MOLLER, C. : The Theory of Relativity, Oxford, Clarendon Press (1952).

[ $\left.{ }^{2}\right]$ SHARMA, J.P. : J. Pure Appl. Geophys. 97 (1972), 14.

['] SHARMA, J.P. : J. Geophys. 78 (23) (1973), 4821.

['] SHARMA, J.P. : : Study of the Internal Structure of a Planet in General Relativity, J. Pure Appl. Maths. (1976, accepted).

$\left[{ }^{5}\right]$ SHARMA, J.P. : J. Geophys. Res. 78 (23) (1973), 31.

DEPARTMENT OF APPLIED SCIENCES

M.M.M. ENGINEERING COLLEGE

GORAKHPUR, INDIA

\section{Ö Z E T}

Bu çalışmada gravitasyonel kütle yoğunluğu sabit olan bir akışkan küreye ait alan denklemlerinin çözümleri elde edilmektedir. 